\title{
'Charm' Strawberry
}

Chad E. Finn ${ }^{2}$

U.S. Department of Agriculture-Agricultural Research Service, Horticultural Crops Research Unit, 3420 NW Orchard Avenue, Corvallis, OR 97330

Patrick P. Moore

Department of Horticulture and Landscape Architecture, Washington State University, Puyallup, WA 98371

Brian M. Yorgey

Department of Food Science and Technology, Oregon State University, Corvallis, OR 97331

Jungmin Lee

U.S. Department of Agriculture-Agricultural Research Service, Horticultural Crops Research Unit Worksite, Parma, ID 83660

Bernadine C. Strik

Department of Horticulture, Oregon State University, Corvallis, OR 97331

\section{Chaim Kempler ${ }^{1}$}

Agriculture and Agri-Food Canada, Pacific Agri-Food Research Centre, Agassiz, British Columbia, Canada VOM 1 AO

\section{Robert R. Martin}

U.S. Department of Agriculture-Agricultural Research Service, Horticultural Crops Research Unit, 3420 NW Orchard Avenue, Corvallis, OR 97330

Additional index words. Fragaria ×ananassa, fruit breeding, short-day, June-bearing, processing

'Charm' is a new June-bearing (short-day) strawberry (Fragaria $\times$ ananassa Duchesne ex Rozier) cultivar from the U.S. Department of Agriculture-Agricultural Research Service (USDA-ARS) breeding program in Corvallis, OR, released in cooperation with the Oregon Agricultural Experiment Station and the Washington State University Agricultural

\footnotetext{
Received for publication 17 June 2013. Accepted for publication 1 July 2013 .

This research was partially funded by the Oregon Strawberry and Washington Strawberry Commissions.

We gratefully acknowledge the assistance of Ted Mackey, Connie Pace, Gil Buller, Nino Adams, and Wendy Hoashi-Erhardt in the evaluation of 'Charm'; Nola Mosier and Kara Sarver for efforts to produce the G1 material that is free of known viruses; Chrislyn Particka (formerly with Sakuma Bros. Farms, Mount Vernon, WA), Arne Goddick (Goddick Farms, Dayton, OR), and Randy Pavlinac (formerly with Sabroso, Woodburn, OR, and Kraemer Farms, Mount Angel, OR) for their commercial evaluation; and Chrislyn Particka and Mike Christenson (Oregon Strawberry Commission) for their coordination of propagation and distribution of plants for trial.

Mention of trade names or commercial products in this manuscript is solely for the purpose of providing specific information and does not imply recommendation or endorsement by the U.S. Department of Agriculture.

${ }^{1}$ Retired.

${ }^{2}$ To whom reprint requests should be addressed; e-mail Chad.Finn@ars.usda.gov.
}

Research Center. 'Charm' is a high-yielding cultivar that produces medium-large sized, easily capped fruit with excellent fruit quality that are particularly suited to ice cream formulations. A U.S. plant patent application (S.N. 13/694,975) has been submitted.

\section{Origin}

'Charm' was selected in 2001 from the cross BC 92-14-31 × WA 94023-1 made in 1999 and was tested as ORUS 2262-2 (Fig. 1). BC 92-14-31 [BC 86-24-107 (Providence $x$ Sumas) $\times$ Nanaimo] was an advanced selection in the Agriculture and Agri-Food Canada breeding program and WA 94023-1 (ORUS $1083-135 \times$ NJ 8713-8) was a selection made in Oregon from a cross made by P. Moore with the Washington State University program (Puyallup). The purpose of the cross was to combine the extremely firm and darkcolored fruit of BC 92-14-31 with the overall good characteristics of WA 94023-1 to improve the latter's firmness and light color. The pedigree represents a mix of genotypes developed in the Pacific Northwest (United States and Canada) and the United Kingdom. 'Charm' was tested most extensively at the Oregon State University-North Willamette Research and Extension Center (Aurora, OR) and the Washington State University Puyallup Research and Extension Center (WSUPuyallup; Puyallup, WA) and in grower fields in Washington and Oregon. The most thorough commercial testing was conducted at Goddik Farms (Dayton, OR), Kraemer Farms (Mount Angel, OR), and Sakuma Bros. Farms (Burlington, WA). At the public research facilities, 'Charm' was planted in multiple replicated trials established from 2002 to 2010.

In all trials, the plants were grown in a matted row system in eight-plant plots with plants initially set $46 \mathrm{~cm}$ apart in the row in Oregon and $38 \mathrm{~cm}$ apart in Washington. The replicated trials had three replications. The plantings were fertilized, renovated, and irrigated using standard commercial practices. Other than two fungicide applications during bloom to control Botrytis fruit rot (Botrytis cinerea Pers.:Fr.), the plantings received no pesticide applications. Fruits were harvested once a week. The average fruit weight for a season was calculated as a weighted mean based on the weight of a randomly selected subsample of 25 fruit from each harvest. In multiple-year trials, yield, average fruit weight, and average fruit rot were analyzed as a split plot in time with cultivar as the main plot and year as the subplot. Fruit firmness was measured in the WSU-Puyallup trial as the force required for a 4-mm-diameter cylinder (Hunter Spring Mechanical Force Gauge Series L; Ametek, Hatfield, PA) to penetrate to a depth of $6 \mathrm{~mm}$ in five randomly selected fruit from each harvest. The average fruit firmness for a season was calculated as a weighted mean. The plantings and the analyses (PROC GLM; SAS Institute, Cary, NC) included the industry standards 'Tillamook' and/or 'Totem' (Finn et al., 2004; Hokanson and Finn, 2000). Plant vigor and fresh fruit characteristics including appearance, firmness, external and internal color, capping (ease with which the calyx was removed), and flavor were rated subjectively at least three times each year in Oregon using a 1 to 9 scale $(1=$ poor vigor, uneven rough appearance, soft fruit, very light-colored, poor separation of calyx from the receptacle, and poor flavor; and $9=$ very vigorous, very uniform and attractive, very firm, dark red, calyx separates easily from the receptacle, and intense flavor, respectively). In multiple years, duplicate subsamples of $\approx 200 \mathrm{~g}$ each were taken randomly from frozen and thawed harvested fruit and were evaluated for soluble solids content, $\mathrm{pH}$, and titratable acidity in the laboratory. Analysis of variance was conducted on the fruit chemistry and subjectively evaluated trait data after checking for normality (PROC UNIVARIATE; SAS Institute). Fruit samples of 'Charm', 'Hood', and 'Totem' were analyzed for the concentration of the anthocyanins using previously described procedures (Lee and Finn, 2007) with a longer high-performance liquid chromatography column (Synergi Hydro-RP $80 \AA$, $250 \mathrm{~mm} \times$ $2 \mathrm{~mm}, 4 \mu \mathrm{m}$; Phenomenex, Inc., Torrance, $\mathrm{CA}$ ). Fruit were also evaluated informally as a thawed, individually quick frozen (IQF) product by growers, processors, and researchers.

\section{Description and Performance}

'Charm' was high-yielding in every trial with yields comparable to or higher than 


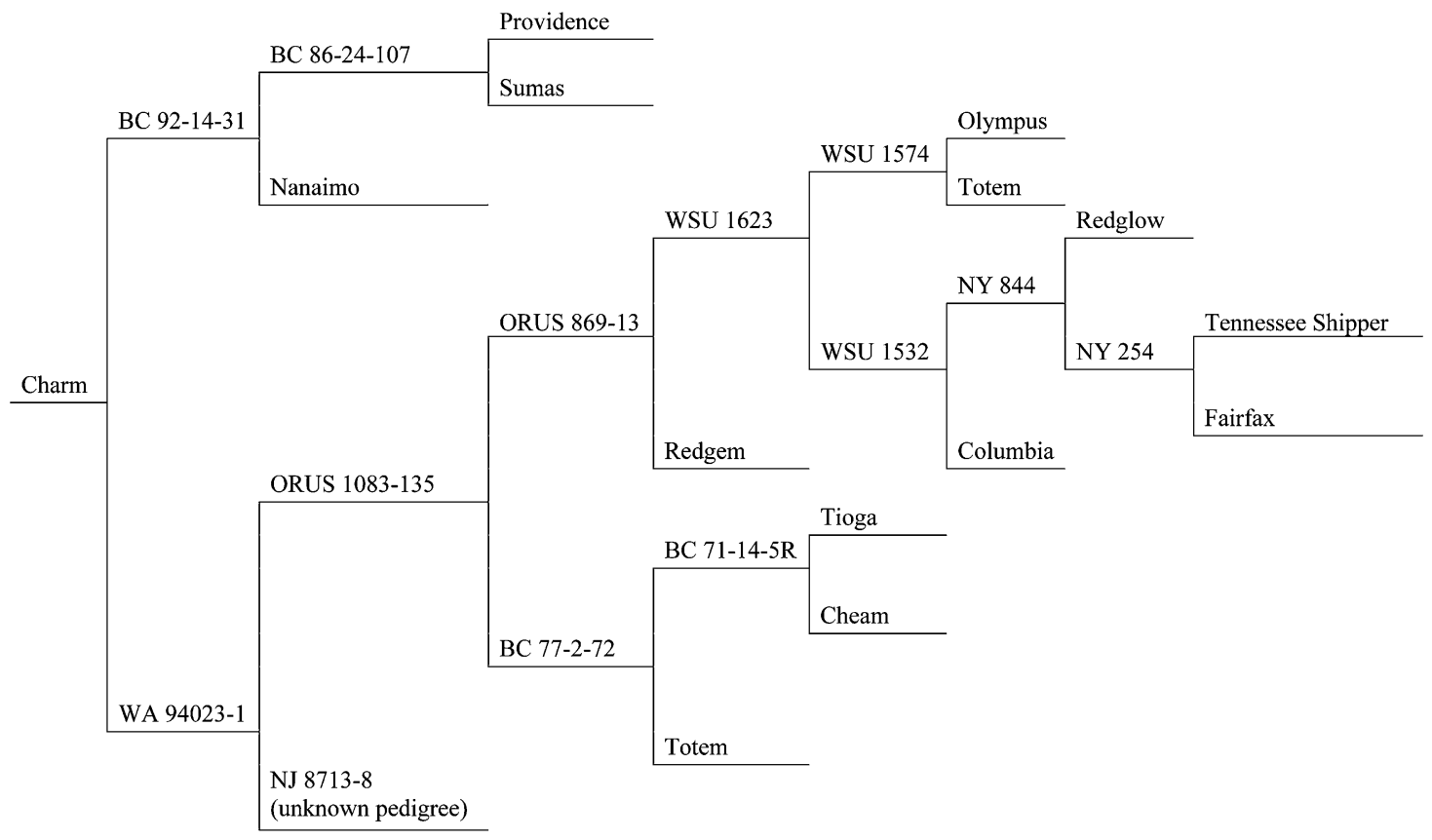

Fig. 1. Pedigree of 'Charm' strawberry; female on top.

other recent releases such as 'Valley Red' and 'Sweet Bliss' or the industry standards, 'Tillamook', 'Totem', and 'Hood' (Tables 1 and 2) (Daubeny et al., 1993; Finn et al., 2004, 2009, 2011; Hokanson and Finn, 2000). In Oregon trials, 'Charm' had the highest mean yield of any of the cultivars evaluated (Table 1). In Washington, although 'Charm' was highyielding in two of three trials, its yields were less than 'Puget Crimson' (Table 2). 'Charm' was comparable in susceptibility to fruit rot (B. cinerea) to 'Totem', 'Tillamook', and 'Hood' in trials in Oregon and Washington (Tables 1 and 2).

'Charm' fruit were typically mediumsized (Fig. 2). 'Charm' was comparable to 'Totem' in fruit weight in all trials, except for the 2008 planted in Oregon and the 2010 Washington-planted trial, where 'Charm' fruit were $\approx 1 \mathrm{~g}$ heavier (Tables 1 and 2 ). 'Charm' fruit were lighter weight than those of 'Tillamook', 'Sweet Bliss', and 'Sweet Sunrise' in the Oregon trials. In Oregon and Washington trials, 'Charm' fruit were lighter weight than those of 'Puget Crimson' (Tables 1 and 2).

'Charm' has excellent overall fruit quality when evaluated as a fresh fruit (Tables 2 and 3). At WSU-Puyallup, where fruit firmness was measured objectively in each trial, 'Charm' fruit had excellent firmness and usually was among the firmest (Table 2). In subjective trials over several years and several plantings in Oregon, 'Charm' fruit were ranked lower than 'Tillamook' but were firmer than 'Puget Reliance' and 'Totem' (Table 3). The fruit were well formed, symmetrical, and attractive and, although not as uniformly shaped as 'Valley Red', they were as good as 'Puget Crimson', 'Sweet Bliss', 'Tillamook', and 'Totem' (Table 3; Fig. 2). Internal and external fruit colors of 'Charm' were acceptable for processing and not as dark as 'Valley Red'. The

Table 1. Yield, fruit weight, and percent fruit rot for 'Charm' and other cultivars in five replicated trials at Oregon State University's North Willamette Research and Extension Center (Aurora, OR).

\begin{tabular}{|c|c|c|c|c|c|c|}
\hline & \multicolumn{2}{|c|}{ Fruit wt $(\mathrm{g})^{\mathrm{z}}$} & \multicolumn{2}{|c|}{ Fruit rot (\%) } & \multicolumn{2}{|c|}{ Yield $\left(\mathrm{kg} \cdot \mathrm{ha}^{-1}\right)$} \\
\hline & Year 1 & Year 2 & Year 1 & Year 2 & Year 1 & Year 2 \\
\hline Cultivar & 2003 & 2004 & 2003 & 2004 & 2003 & 2004 \\
\hline \multicolumn{7}{|l|}{2002 planted } \\
\hline Charm & $14.4 \mathrm{a}$ & $13.8 \mathrm{a}$ & $3.8 \mathrm{~b}$ & $21.6 \mathrm{~b}$ & $39,274 \mathrm{a}$ & $13,559 \mathrm{a}$ \\
\hline Totem & $18.8 \mathrm{a}$ & $10.7 \mathrm{~b}$ & $0.0 \mathrm{a}$ & $6.3 \mathrm{a}$ & $30,986 \mathrm{a}$ & 7,549 a \\
\hline 2003 planted & 2004 & 2005 & 2004 & 2005 & 2004 & 2005 \\
\hline Charm & $17.9 \mathrm{a}$ & $11.2 \mathrm{c}$ & $8.5 \mathrm{a}$ & $4.2 \mathrm{c}$ & $46,851 \mathrm{ab}$ & $29,274 \mathrm{a}$ \\
\hline Pinnacle & $17.8 \mathrm{a}$ & $15.1 \mathrm{ab}$ & $4.6 \mathrm{a}-\mathrm{c}$ & $12.5 \mathrm{a}$ & $48,495 \mathrm{a}$ & $20,339 \mathrm{bc}$ \\
\hline Puget Reliance & $17.8 \mathrm{a}$ & $10.0 \mathrm{c}$ & $4.0 \mathrm{bc}$ & $8.4 \mathrm{ab}$ & $35,946 \mathrm{~cd}$ & $28,927 \mathrm{a}$ \\
\hline Stolo & $16.8 \mathrm{a}$ & $9.9 \mathrm{c}$ & $7.4 \mathrm{ab}$ & $4.9 \mathrm{ab}$ & $38,874 \mathrm{bc}$ & $21,823 \mathrm{bc}$ \\
\hline Sweet Sunrise & $17.7 \mathrm{a}$ & $13.1 \mathrm{a}-\mathrm{c}$ & $3.0 \mathrm{c}$ & $4.6 \mathrm{c}$ & $43,714 \mathrm{a}-\mathrm{c}$ & $25,196 \mathrm{ab}$ \\
\hline Tillamook & $17.6 \mathrm{a}$ & $15.2 \mathrm{a}$ & $4.0 \mathrm{bc}$ & $9.4 \mathrm{ab}$ & $47,482 \mathrm{ab}$ & $20,895 \mathrm{bc}$ \\
\hline Totem & $16.1 \mathrm{a}$ & $9.3 \mathrm{c}$ & $4.5 \mathrm{a}-\mathrm{c}$ & $6.3 \mathrm{ab}$ & $28,577 \mathrm{~d}$ & $18,177 \mathrm{c}$ \\
\hline 2008 planted & 2009 & 2010 & 2009 & 2010 & 2009 & 2010 \\
\hline Charm & $13.6 \mathrm{c}$ & $10.3 \mathrm{~cd}$ & $13.6 \mathrm{a}$ & $19.4 \mathrm{a}-\mathrm{c}$ & 34,152 a & $26,880 \mathrm{a}$ \\
\hline Puget Crimson & $13.1 \mathrm{c}$ & $13.5 \mathrm{~b}$ & $11.9 \mathrm{ab}$ & $20.3 \mathrm{ab}$ & $28,721 \mathrm{ab}$ & $15,475 \mathrm{c}$ \\
\hline Sweet Bliss & $16.3 \mathrm{~b}$ & $14.1 \mathrm{ab}$ & $16.9 \mathrm{a}$ & $23.6 \mathrm{a}$ & $34,561 \mathrm{a}$ & $21,429 \mathrm{a}-\mathrm{c}$ \\
\hline Sweet Sunrise & $17.0 \mathrm{~b}$ & $13.0 \mathrm{bc}$ & $10.2 \mathrm{ab}$ & $12.2 \mathrm{~cd}$ & $33,713 \mathrm{a}$ & $23,781 \mathrm{ab}$ \\
\hline Tillamook & $20.0 \mathrm{a}$ & $16.6 \mathrm{a}$ & $6.0 \mathrm{~b}$ & $11.7 \mathrm{~d}$ & $29,679 \mathrm{a}$ & $21,267 \mathrm{a}-\mathrm{c}$ \\
\hline Totem & $15.1 \mathrm{bc}$ & $9.5 \mathrm{~d}$ & $16.2 \mathrm{a}$ & $14.5 \mathrm{~b}-\mathrm{d}$ & $23,477 \mathrm{~b}$ & $17,689 \mathrm{bc}$ \\
\hline Valley Red & $14.5 \mathrm{bc}$ & $12.7 \mathrm{bc}$ & $6.0 \mathrm{~b}$ & $14.0 \mathrm{~b}-\mathrm{d}$ & $29,439 \mathrm{a}$ & $24,133 \mathrm{ab}$ \\
\hline 2009 planted & 2010 & 2011 & 2010 & 2011 & 2010 & 2011 \\
\hline Charm & $16.8 \mathrm{ab}$ & $12.3 \mathrm{~b}$ & $18.3 \mathrm{ab}$ & $28.9 \mathrm{ab}$ & $43,822 \mathrm{a}$ & $28,925 \mathrm{a}$ \\
\hline Puget Crimson & $17.6 \mathrm{ab}$ & $12.9 \mathrm{ab}$ & $13.5 \mathrm{~b}$ & $36.9 \mathrm{ab}$ & $33,669 \mathrm{a}$ & $15,789 \mathrm{~b}$ \\
\hline Tillamook & $18.2 \mathrm{a}$ & $14.9 \mathrm{a}$ & $24.2 \mathrm{a}$ & $44.0 \mathrm{a}$ & $41,063 \mathrm{a}$ & $22,417 \mathrm{ab}$ \\
\hline Totem & $15.0 \mathrm{~b}$ & $11.8 \mathrm{~b}$ & $11.1 \mathrm{~b}$ & $25.8 \mathrm{~b}$ & $22,656 \mathrm{~b}$ & $20,680 \mathrm{ab}$ \\
\hline 2010 planted & 2011 & 2012 & 2011 & 2012 & 2011 & 2012 \\
\hline Charm & $17.7 \mathrm{a}$ & $11.9 \mathrm{a}$ & $27.6 \mathrm{a}$ & $16.8 \mathrm{~b}$ & $39,834 \mathrm{a}$ & $25,226 \mathrm{a}$ \\
\hline Tillamook & $14.9 \mathrm{~b}$ & $11.2 \mathrm{a}$ & $22.2 \mathrm{a}$ & $41.1 \mathrm{a}$ & $31,885 \mathrm{a}$ & $6,732 \mathrm{c}$ \\
\hline Totem & $16.2 \mathrm{ab}$ & $11.8 \mathrm{a}$ & $23.5 \mathrm{a}$ & $29.4 \mathrm{a}$ & $18,577 \mathrm{~b}$ & $17,242 \mathrm{~b}$ \\
\hline
\end{tabular}

${ }^{\mathrm{z}}$ Means within a column followed by the same letter are not significantly different, $P>0.05$, by least significant difference test.

fruit capped extremely well and were rated easier to cap than 'Tillamook' and 'Sweet Bliss' (Table 3). 'Charm' fruit had an excellent fresh strawberry flavor that was not rated as highly as the outstanding-flavored 'Puget Crimson' but was comparable to 'Sweet Bliss' and better than 'Puget Reliance', 'Tillamook', 'Totem', and 'Valley Red' (Table 3).

As part of the breeding program, thawed, IQF fruit of each genotype were evaluated by an expert panel of researchers and industry members annually in the off-season. The 
Table 2. Yield, fruit weight, and fruit firmness and percent fruit rot for 'Charm' and other cultivars in five replicated trials at Washington State UniversityPuyallup Research and Extension Center.

\begin{tabular}{|c|c|c|c|c|c|c|c|c|}
\hline \multirow[b]{2}{*}{ Cultivar } & \multicolumn{2}{|c|}{ Fruit wt $(\mathrm{g})^{\mathrm{z}}$} & \multicolumn{2}{|c|}{ Fruit rot $(\%)$} & \multicolumn{2}{|c|}{ Fruit firmness $(\mathrm{g})$} & \multicolumn{2}{|c|}{ Yield $\left(\mathrm{kg} \cdot \mathrm{ha}^{-1}\right)$} \\
\hline & Year 1 & Year 2 & Year 1 & Year 2 & Year 1 & Year 2 & Year 1 & Year 2 \\
\hline 2009 planted & 2010 & 2011 & 2010 & 2011 & 2010 & 2011 & 2010 & 2011 \\
\hline Charm & $15.2 \mathrm{a}-\mathrm{c}$ & $9.0 \mathrm{~b}-\mathrm{d}$ & $13.5 \mathrm{ab}$ & $12.6 \mathrm{ab}$ & $237 \mathrm{a}$ & $242 \mathrm{a}$ & $40,350 \mathrm{ab}$ & $32,595 \mathrm{ab}$ \\
\hline Hood & $11.4 \mathrm{c}$ & $5.6 \mathrm{~d}$ & $15.4 \mathrm{ab}$ & $22.2 \mathrm{ab}$ & $218 \mathrm{a}$ & $193 \mathrm{bc}$ & $14,753 \mathrm{c}$ & $3,495 \mathrm{c}$ \\
\hline Puget Crimson & $15.8 \mathrm{a}-\mathrm{c}$ & $14.6 \mathrm{a}$ & $10.5 \mathrm{ab}$ & $9.0 \mathrm{ab}$ & $170 \mathrm{~b}$ & $192 \mathrm{bc}$ & $30,798 \mathrm{a}$ & $45,448 \mathrm{a}$ \\
\hline Puget Reliance & $14.2 \mathrm{a}-\mathrm{c}$ & $9.5 \mathrm{~b}-\mathrm{d}$ & $5.6 \mathrm{~b}$ & $8.2 \mathrm{ab}$ & $219 \mathrm{a}$ & $172 \mathrm{c}$ & $16,193 \mathrm{bc}$ & $22,936 \mathrm{bc}$ \\
\hline Puget Summer & $13.3 \mathrm{bc}$ & $9.2 \mathrm{~b}-\mathrm{d}$ & $17.7 \mathrm{a}$ & $25.0 \mathrm{a}$ & $219 a$ & $250 \mathrm{a}$ & $8,209 \mathrm{c}$ & $8,405 \mathrm{c}$ \\
\hline Tillamook & $18.9 \mathrm{a}$ & $13.6 \mathrm{ab}$ & $9.2 \mathrm{ab}$ & $17.7 \mathrm{ab}$ & $219 \mathrm{a}$ & $209 a-c$ & $18,537 \mathrm{c}$ & $11,736 \mathrm{c}$ \\
\hline Totem & $11.3 \mathrm{c}$ & $8.3 \mathrm{~cd}$ & $9.8 \mathrm{ab}$ & $7.7 \mathrm{ab}$ & $247 \mathrm{a}$ & $222 \mathrm{ab}$ & $15,064 \mathrm{bc}$ & $18,100 \mathrm{bc}$ \\
\hline Valley Red & $17.2 \mathrm{ab}$ & $12.6 \mathrm{a}-\mathrm{c}$ & $10.0 \mathrm{ab}$ & $5.0 \mathrm{~b}$ & $213 a b$ & $212 \mathrm{a}-\mathrm{c}$ & $18,382 \mathrm{ab}$ & $32,286 \mathrm{ab}$ \\
\hline 2010 planted & 2011 & 2012 & 2011 & 2012 & 2011 & 2012 & 2011 & 2012 \\
\hline Charm & $14.9 \mathrm{ab}$ & $9.0 \mathrm{~b}$ & $8.6 \mathrm{a}$ & $16.1 \mathrm{a}$ & $253 \mathrm{a}$ & $312 \mathrm{a}$ & $23,312 \mathrm{a}$ & $18,967 \mathrm{ab}$ \\
\hline Hood & $11.9 \mathrm{~b}$ & $8.8 \mathrm{~b}$ & $12.5 \mathrm{a}$ & $15.3 \mathrm{a}$ & $185 \mathrm{~b}$ & $226 b-d$ & $25,235 \mathrm{a}$ & $14,063 \mathrm{~b}$ \\
\hline Puget Summer & $13.3 \mathrm{~b}$ & $12.1 \mathrm{ab}$ & $9.6 \mathrm{a}$ & $18.8 \mathrm{a}$ & $222 \mathrm{ab}$ & $234 \mathrm{bc}$ & $16,323 \mathrm{a}$ & $19,687 \mathrm{ab}$ \\
\hline Puget Crimson & $19.8 \mathrm{a}$ & $19.5 \mathrm{a}$ & $10.3 \mathrm{a}$ & $15.9 \mathrm{a}$ & 192 b & $179 \mathrm{~d}$ & $25,203 \mathrm{a}$ & $37,694 \mathrm{a}$ \\
\hline Puget Reliance & $11.4 \mathrm{~b}$ & $10.0 \mathrm{~b}$ & $6.1 \mathrm{a}$ & $14.8 \mathrm{a}$ & $182 \mathrm{~b}$ & $206 \mathrm{~cd}$ & $29,389 \mathrm{a}$ & $25,307 \mathrm{ab}$ \\
\hline Tillamook & $18.0 \mathrm{a}$ & $13.0 \mathrm{ab}$ & $9.4 \mathrm{a}$ & $9.6 \mathrm{a}$ & $231 \mathrm{ab}$ & $255 \mathrm{bc}$ & $21,591 \mathrm{a}$ & $20,416 a b$ \\
\hline Totem & $12.0 \mathrm{~b}$ & $10.4 \mathrm{~b}$ & $9.8 \mathrm{a}$ & $16.8 \mathrm{a}$ & $198 \mathrm{~b}$ & $268 \mathrm{ab}$ & $23,796 \mathrm{a}$ & $27,955 \mathrm{ab}$ \\
\hline 2011 planted & 2012 & & 2012 & & 2012 & & 2012 & \\
\hline Charm & $13.0 \mathrm{~b}$ & - & $17.6 \mathrm{a}$ & - & $291 \mathrm{a}$ & - & $32,954 \mathrm{a}$ & - \\
\hline Hood & $8.6 \mathrm{c}$ & - & $8.4 \mathrm{ab}$ & - & $255 \mathrm{ab}$ & - & $7,538 \mathrm{~d}$ & - \\
\hline Nisga’a & $11.0 \mathrm{bc}$ & - & $17.5 \mathrm{a}$ & - & $265 \mathrm{ab}$ & - & $20,077 \mathrm{a}-\mathrm{d}$ & - \\
\hline Puget Crimson & $18.2 \mathrm{a}$ & - & $7.1 \mathrm{ab}$ & - & $224 \mathrm{~b}$ & - & $29,368 \mathrm{ab}$ & - \\
\hline Puget Reliance & $13.3 \mathrm{~b}$ & - & $8.4 \mathrm{ab}$ & - & $207 \mathrm{~b}$ & - & $23,384 \mathrm{a}-\mathrm{c}$ & - \\
\hline Puget Summer & $13.9 \mathrm{~b}$ & - & $6.4 \mathrm{~b}$ & - & $288 \mathrm{a}$ & - & $12,012 \mathrm{~cd}$ & - \\
\hline Totem & $11.1 \mathrm{bc}$ & 一 & $10.3 \mathrm{ab}$ & - & $260 \mathrm{ab}$ & 一 & $23,784 \mathrm{a}-\mathrm{c}$ & 一 \\
\hline
\end{tabular}

${ }^{\mathrm{z}}$ Means within a column followed by the same letter are not significantly different, $P>0.05$, by least significant difference test.

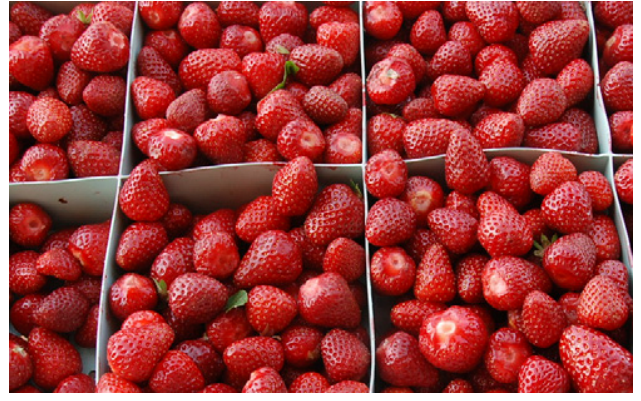

Fig. 2. Capped, harvested fruit of 'Charm' strawberry for processing.

Table 3. Mean scores over 8 years for subjectively evaluated characteristics in the field for 'Charm' and six other strawberry cultivars planted at Oregon State University's North Willamette Research and Extension Center (Aurora, OR).

\begin{tabular}{lccccccc}
\hline & \multicolumn{7}{c}{ Fresh fruit characteristics } \\
\cline { 2 - 6 } Cultivar & Plant vigor $^{2}$ & Appearance & Firmness & External & Internal & Capping & Flavor \\
\hline Charm & $8.6 \mathrm{a}^{\mathrm{y}}$ & $7.6 \mathrm{bc}$ & $7.7 \mathrm{ab}$ & $7.2 \mathrm{bc}$ & $7.1 \mathrm{bc}$ & $8.1 \mathrm{a}$ & $7.4 \mathrm{~b}$ \\
Puget Crimson & $7.7 \mathrm{~b}$ & $7.1 \mathrm{c}$ & $7.7 \mathrm{ab}$ & $7.7 \mathrm{a}$ & $7.3 \mathrm{bc}$ & $8.0 \mathrm{a}$ & $7.8 \mathrm{a}$ \\
Puget Reliance & $7.3 \mathrm{bc}$ & $8.1 \mathrm{a}$ & $5.9 \mathrm{~d}$ & $7.0 \mathrm{c}$ & $6.5 \mathrm{~d}$ & $8.1 \mathrm{a}$ & $6.8 \mathrm{c}$ \\
Sweet Bliss & $7.3 \mathrm{bc}$ & $7.9 \mathrm{ab}$ & $7.7 \mathrm{ab}$ & $7.2 \mathrm{bc}$ & $7.0 \mathrm{c}$ & $7.3 \mathrm{~b}$ & $7.6 \mathrm{ab}$ \\
Tillamook & $6.9 \mathrm{c}$ & $7.1 \mathrm{c}$ & $8.2 \mathrm{a}$ & $7.5 \mathrm{ab}$ & $7.2 \mathrm{bc}$ & $7.3 \mathrm{~b}$ & $6.7 \mathrm{c}$ \\
Totem & $7.3 \mathrm{bc}$ & $7.3 \mathrm{c}$ & $7.0 \mathrm{c}$ & $7.5 \mathrm{ab}$ & $7.4 \mathrm{ab}$ & $7.7 \mathrm{ab}$ & $6.9 \mathrm{c}$ \\
Valley Red & $7.5 \mathrm{~b}$ & $8.3 \mathrm{a}$ & $7.3 \mathrm{bc}$ & $7.8 \mathrm{a}$ & $7.6 \mathrm{a}$ & $8.2 \mathrm{a}$ & $6.9 \mathrm{c}$ \\
\hline
\end{tabular}

${ }^{\mathrm{z}}$ Traits scored on a 1 to 9 scale: 1 = poor vigor, uneven rough appearance, soft fruit, very light-colored, poor separation of calyx from the receptacle ("capping"), and poor flavor; and 9 = very vigorous, very uniform and attractive, very firm, dark red, calyx separates easily from the receptacle, and intense flavor, respectively. ${ }^{\mathrm{y}}$ Means within a column followed by the same letter are not significantly different, $P>0.05$, by least significant difference test.

processors, particularly those who sell to ice cream manufacturers, are anxious to identify selections that could complement or replace 'Hood' and 'Totem', which are the standards

for the ice cream industry. As a selection in 2007, 'Charm' was identified by the industry as among the top five with potential as an ingredient for ice cream formulations. In
2008 , in an in-house evaluation by a major ice cream manufacturer, 'Charm' was identified as being "very impressive" and "one with potential as a "Hood' replacement." The fruit chemistry values for 'Charm' were good and acceptable for commercial processing (Table 4). The soluble solids content was not as high as for 'Hood' or 'Puget Crimson' but was comparable to 'Totem' and the other cultivars. Ideally fruit for processing have a $\mathrm{pH}$ below 3.50; 'Charm' fruit had a $\mathrm{pH}$ of 3.42 , comparable to other cultivars (Table 4 ) (Wrolstad et al., 2008). 'Charm' fruit had an acceptable titratable acidity, higher than 'Pinnacle' but similar to the other cultivars in the trial (Table 4). The anthocyanin profile of 'Charm' fruit was similar to 'Hood' or 'Totem' profiles: glycosides of pelargonidin and cyanidin, with pelargonidin-3-glucoside being the main pigment (Table 5). The concentrations of the five strawberry anthocyanins were comparable between 'Charm' and the two standards for the ice cream industry, 'Totem' and 'Hood'. 'Charm' tentatively contained a higher proportion of acylated anthocyanin ( $25 \%$ of total) than for 'Hood' $(23 \%)$ or 'Totem' (18\%). 'Totem' and 'Hood', with $86 \%$ and $87 \%$, respectively, appeared to have a slightly higher proportion of pelargonidinvs. cyanidin-based anthocyanins than did 'Charm' (82\%).

'Charm' fruit consistently ripened with 'Totem', later than 'Sweet Sunrise', and earlier than 'Puget Crimson' in Oregon (Table 6). The trend was similar in Washington, although 'Charm' ripened almost 1 week earlier than 'Tillamook', whereas in Oregon, there was only a couple days difference in the $50 \%$ ripe date. 'Charm' ripened later than 'Hood' (data not shown). For all cultivars in common 
Table 4. Percent soluble solids, $\mathrm{pH}$, and titratable acidity for fruit purees of eleven strawberry cultivars grown at Oregon State University's North Willamette Research and Extension Center (Aurora, OR) from 2004 to 2011.

\begin{tabular}{lccc}
\hline Cultivar & Percent soluble solids $\left({ }^{\circ} \text { Brix }\right)^{\mathrm{z}}$ & $\mathrm{pH}$ & Titratable acidity $\left(\mathrm{g} \cdot \mathrm{L}^{-1}\right.$ as citric acid) \\
\hline Charm & $7.65 \mathrm{c}$ & $3.42 \mathrm{bc}$ & $9.45 \mathrm{a}-\mathrm{c}$ \\
Hood & $10.39 \mathrm{a}$ & $3.51 \mathrm{ab}$ & $8.38 \mathrm{~cd}$ \\
Pinnacle & $7.88 \mathrm{c}$ & $3.57 \mathrm{a}$ & $7.45 \mathrm{~d}$ \\
Puget Crimson & $9.54 \mathrm{ab}$ & $3.35 \mathrm{c}$ & $10.52 \mathrm{ab}$ \\
Puget Reliance & $8.23 \mathrm{c}$ & $3.41 \mathrm{bc}$ & $9.14 \mathrm{~b}-\mathrm{d}$ \\
Shuksan & $7.98 \mathrm{c}$ & $3.47 \mathrm{a}-\mathrm{c}$ & $9.83 \mathrm{a}-\mathrm{c}$ \\
Stolo & $8.50 \mathrm{c}$ & $3.46 \mathrm{a}-\mathrm{c}$ & $8.40 \mathrm{~cd}$ \\
Sweet Bliss & $7.94 \mathrm{c}$ & $3.36 \mathrm{bc}$ & $11.10 \mathrm{a}$ \\
Tillamook & $7.75 \mathrm{c}$ & $3.47 \mathrm{a}-\mathrm{c}$ & $8.69 \mathrm{~b}-\mathrm{d}$ \\
Totem & $8.69 \mathrm{bc}$ & $3.51 \mathrm{ab}$ & $8.76 \mathrm{~b}-\mathrm{d}$ \\
Valley Red & $7.70 \mathrm{c}$ & $3.49 \mathrm{a}-\mathrm{c}$ & $8.37 \mathrm{~cd}$ \\
\hline
\end{tabular}

${ }^{2}$ Means within a column followed by the same letter are not significantly different, $P>0.05$, by least significant difference test.

Table 5. Anthocyanin concentrations ( $\mathrm{mg}$ of cyanidin-3-glucoside/100 g) of fruit 'Charm' and two standard strawberry cultivars harvested in 2012 from trial at Oregon State University's North Willamette Research and Extension Center (Aurora, OR). ${ }^{\mathrm{z}}$

\begin{tabular}{|c|c|c|c|c|c|c|c|c|c|c|c|}
\hline \multirow{2}{*}{$\frac{\text { Cultivar }}{\text { Charm }}$} & \multicolumn{2}{|c|}{ Cyanidin-3-glucoside } & \multicolumn{2}{|c|}{ Pelargonidin-3-glucoside } & \multicolumn{2}{|c|}{ Pelargonidin-3-rutinoside } & \multicolumn{2}{|c|}{ Cyanidin-3-glucoside-malonate } & \multicolumn{2}{|c|}{$\begin{array}{c}\text { Pelargonidin-3-glucoside } \\
\text { malonate }\end{array}$} & \multirow{2}{*}{$\frac{\text { Total }}{59.6}$} \\
\hline & 6.1 & 10 & 33.5 & 56 & 5.3 & 9 & 4.4 & 7 & 10.3 & 17 & \\
\hline Totem & 4.3 & 7 & 38.6 & 67 & 4.1 & 7 & 3.6 & 6 & 6.9 & 12 & 57.5 \\
\hline Hood & 4.7 & 7 & 41.5 & 62 & 4.7 & 7 & 4.0 & 6 & 11.6 & 17 & 66.5 \\
\hline
\end{tabular}

${ }^{2}$ Anthocyanins are listed in the order of high-performance liquid chromatography elution. Values in italics are proportions of the total anthocyanins.

Table 6. Dates when harvest passed 5\%,50\%, and 95\% of total yield and the length of the harvest season for 'Charm' and other cultivars picked in the same year from trials at Oregon State University's North Willamette Research (OSU-NWREC) and Extension Center (Aurora, OR) and at Washington State University (WSU)-Puyallup Research and Extension Center.

\begin{tabular}{|c|c|c|c|c|c|}
\hline & \multicolumn{4}{|c|}{ Harvest season } & \multirow[b]{2}{*}{ Length of harvest season (d } \\
\hline & No. ${ }^{z}$ & $5 \%$ & $50 \%$ & $95 \%$ & \\
\hline Stolo & 2 & 30 May & 6 June & 18 June & 19 \\
\hline Sweet Sunrise & 3 & 27 May & 6 June & 19 June & 23 \\
\hline Charm & 6 & 7 June & 14 June & 23 June & 16 \\
\hline Totem & 6 & 6 June & 14 June & 24 June & 18 \\
\hline Tillamook & 6 & 8 June & 16 June & 27 June & 19 \\
\hline Valley Red & 2 & 11 June & 19 June & 1 July & 20 \\
\hline Hood & 5 & 10 June & 21 June & 4 July & 24 \\
\hline Charm & 5 & 13 June & 23 June & 4 July & 21 \\
\hline Puget Reliance & 5 & 13 June & 24 June & 7 July & 24 \\
\hline Totem & 5 & 13 June & 25 June & 6 July & 23 \\
\hline Tillamook & 4 & 17 June & 29 June & 11 July & 24 \\
\hline Puget Summer & 4 & 21 June & 1 July & 11 July & 20 \\
\hline
\end{tabular}

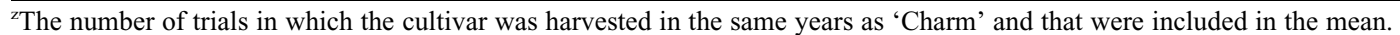

between the Washington and Oregon trials, the harvest season was longer in Washington (Table 6). In Oregon, 'Charm' typically had the shortest season from 5\% to $95 \%$ harvest, $\approx 16$ d. In Washington, 'Valley Red', 'Puget Summer', and 'Charm' had harvest seasons of 10 to $21 \mathrm{~d}$, whereas for 'Totem' and 'Tillamook', they were 23 to $24 \mathrm{~d}$.

'Charm' has been one of the most vigorous selections ever evaluated in the USDAARS program (Fig. 3; Table 3). The plants were more vigorous than all of the other cultivars trialed (Table 3 ). One of the critical concerns evaluated when 'Charm' was in grower trials was whether its vigor negatively affected harvest efficiency. Picking crew supervisors, who oversaw the commercial harvesting of 'Charm', felt that this cultivar could be harvested efficiently, because the fruit could be spotted and capped easily by their picking crews (R. Pavlinac, personal communication). Although not screened for any particular diseases, the plants held up well through the second harvest season and appear to have good virus tolerance. Under our minimal spray program, 'Charm' did not show any particular susceptibility to pests. 'Charm' seems to be a particularly durable plant holding up well across challenging years and grower field situations. In bench screening tests conducted by Agriculture and Agri-Foods Canada, 'Charm' was found to be susceptible to Phytophthora fragariae Hickman races Cdn4 and Cdn-5 and not surprisingly lacked the Rpf1 simple sequence repeat marker indicative of resistance to these races (A. Jamieson and $\mathrm{M}$. Mathey, personal communication).

'Charm' should be grown by commercial growers producing strawberries in a perennial matted row production system for processing markets, particularly those selling to processors who prepare ice cream formulations. This cultivar is high-yielding and vigorous with very uniformly shaped, medium- to large-sized fruit that have outstanding quality in processed applications.

\section{Availability}

A plant patent application has been submitted. The nuclear stock plants for propagation have tested negative for Apple mosaic Tomato ringspot, Strawberry mild yellow edge, Tobacco streak, and Strawberry necrotic shock viruses by enzyme-linked immunosorbent assay and negative for Strawberry mottle, Strawberry veinbanding, Strawberry crinkle, Strawberry pallidosis, Strawberry latent ringspot, Beet pseudo yellows, and Fragaria chiloensis latent viruses in reverse 


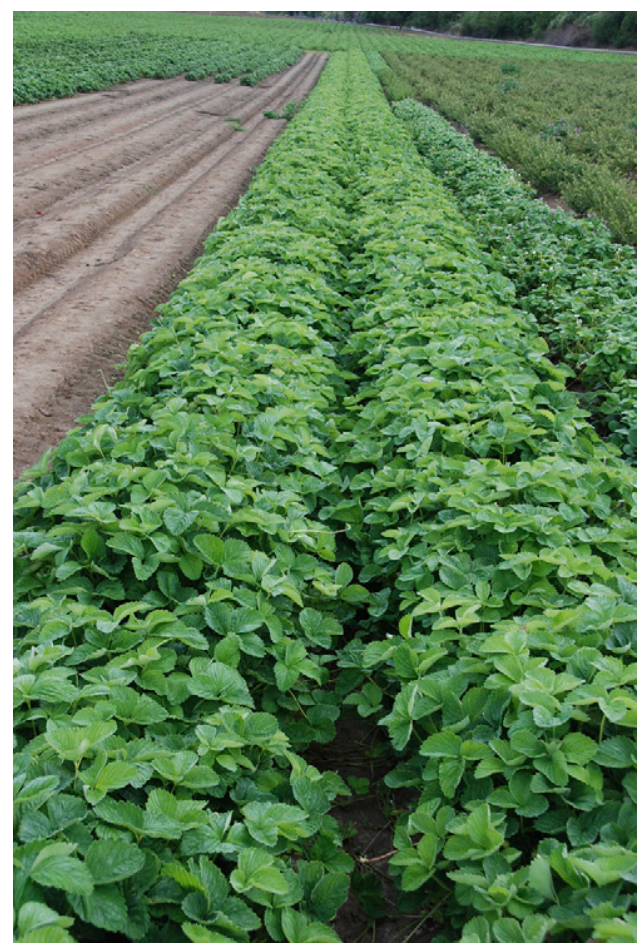

Fig. 3. Plants of 'Charm' strawberry in a grower trial, June 2011, Mount Angel, OR.

transcription-polymerase chain reaction assays, phytoplasmas in polymerase chain reaction assays, and have indexed negative when grafted onto $F$. vesca L. Further information on licensing or a list of nurseries propagating 'Charm' are available on written request to C. Finn as are contact information for commercial laboratories that are able to genetically fingerprint vegetative tissue to determine whether a genotype is 'Charm'. The USDA-ARS does not have commercial quantities of plants to distribute. In addition, plants of this release have been deposited in the National Plant Germplasm System, accession number CFRA 2119.001 (PI 664911), where they will be available for research purposes, including development of new cultivars.

\section{Literature Cited}

Daubeny, H.A., F.J. Lawrence, and P.P. Moore. 1993. 'Totem' strawberry. Fruit Var. J. 47:182184.

Finn, C.E., C. Kempler, P.P. Moore, B.C. Strik, B.M. Yorgey, R.R. Martin, and G.J. Galletta. 2011. 'Sweet Bliss' strawberry. HortScience 46:1701-1705.

Finn, C.E., P.P. Moore, C. Kempler, B.M. Yorgey, B.C. Strik, and R.R. Martin. 2009. 'Valley Red' strawberry. HortScience 44:1468-1471.

Finn, C.E., B. Yorgey, B.C. Strik, and P.P. Moore. 2004. 'Tillamook' and 'Pinnacle' strawberries. HortScience 39:1487-1489.

Hokanson, S.C. and C.E. Finn. 2000. Strawberry cultivar use in North America. HortTechnology 10:94-106.

Lee, J. and C.E. Finn. 2007. Anthocyanins and other polyphenolics in American elderberry (Sambucus canadensis) and European elderberry (S. nigra) cultivars. J. Sci. Food Agr. 87:2665-2675.

Wrolstad, R.E., T. Ngo, C.E. Finn, and Y. Zhao. 2008. Color quality of fresh and processed strawberries. ACS Symp. Ser. 983:18-42. 